Article

\title{
Evaluation of the Activity and Tolerability of a Cosmetic Treatment for the Periocular Area on the Aging Face: Controlled Clinical and Instrumental Evaluation vs. Placebo
}

\author{
Adele Sparavigna ${ }^{1, \dagger}, *$, Beatrice Tenconi ${ }^{1, \dagger}$, Ileana De Ponti ${ }^{1, \dagger}$ and Giancarlo Guglielmini ${ }^{2}$ \\ 1 DermIng S.r.l. Clinical Research Institute, Viale Cesare Battisti 38, 20900 Monza, Italy; \\ E-Mails: beatrice.tenconi@derming.com (B.T.); ileana.deponti@derming.com (I.P.) \\ 2 Sinerga SpA, Via della Pacciarna 67, 21050 Gorla Maggiore, Italy; \\ E-Mail: g.guglielmini@sinerga.it
}

$\dagger$ These authors contributed equally to this work.

* Author to whom correspondence should be addressed; E-Mail: adele.sparavigna@derming.com; Tel.: +39-039-329-666; Fax: +39-039-232-9448.

Received: 7 February 2014; in revised form: 13 May 2014 / Accepted: 13 May 2014 /

Published: 21 May 2014

\begin{abstract}
The aim of this study was to evaluate clinically and by non invasive instrumental evaluations the efficacy and tolerability of a cosmetic treatment containing an innovative active ingredient, ethyl ximenynate, in the prevention of dark circles under the eyes and blemishes of skin aging on the eye profile. Evaluations were performed at baseline and after 2 and 4 weeks of treatment on 22 volunteers; the study was planned as a double blind half-face trial. Data showed a trend towards a lifting of the eyelid skin; all investigator scores (eye puffiness, skin smoothness, roughness and dryness) decreased. In addition, profilometric results confirmed the anti-wrinkles efficacy of the active product and its lifting action, while those related to spectrophotometry underlined the decongestant effect on dark circles that appeared, at the end of the study, to be less marked. The improvement of skin microcirculation and capillary resistance and vascular tone in the eye area underlines a general mitigation of all relevant skin imperfections and blemishes of skin aging on the eye profile. The active ingredient was shown to be effective for the cosmetic treatment of the periocular area.
\end{abstract}

Keywords: circles under the eye; periocular wrinkles; eye profile; ethyl ximenynate 


\section{Introduction}

Midfacial aging is a natural process that affects every anatomic component of the midface. Tissue descent, fat atrophy, and deterioration in the condition and appearance of the skin are the main aesthetically undesirable changes that occur with aging. The skin loses its sheen and becomes more sallow, pigmented, coarse and wrinkled. Midfacial aging is directly related to changes that occur in the cheek and in the periocular region, a specific area of the skin characterized by micro-anatomic features which makes it different from the surrounding facial skin. The epidermis is thinner and very sensitive, characterized by a reduced barrier function and by an abnormal hydrolipidic layer; the dermis is also thinner with a reduced collagen and elastin content which makes the skin less tense and more prone to becoming slack and relaxed. This may cause a decreased blood and lymph flow resulting in fluid accumulation and pseudo-edema with further progression of the extrusion of the lower eyelid. From a clinical point of view, the area may have discoloration (yellowish or brownish hue), edema, wrinkles and ptosis of the eyelid. Treatment of the periocular area should be oriented to the reduction of predisposing factors (smoke, free radicals, UV exposure) and improvement of skin microcirculation, both for vascular and lymphatic compartments $[1,2]$.

In some reviews, ethyl ximenynate has been described as a high performance microcirculation modulator effective in improving the superficial blood vessels flow and in increasing blood perfusion, and therefore ideal for treatments where modification of microcirculation is indicated. Ethyl ximenynate is a semisynthetic fluid oil obtained from the esterification of ximeninic acid, a long chain fatty acid, with a double and triple bond (11-octandecen-9-ioic acid), purified from the plant Olax dissitiflora. Its usage in cosmetics is suggested to ameliorate the circulatory disease of lower extrimities, in particular when an alteration of the capillary permeability is present, such as heavy lower extremities, body firming and cellulite reduction [3-9].

In this study, the cosmetic use for ethyl ximenynate was investigated for the prevention of circles under the eyes and blemishes of skin aging on the eye profile.

\section{Experimental Section}

\subsection{Formulation}

The formulation is based on an active ingredient at 3\% concentrated (International Nomenclature of Cosmetic Ingredients: ethyl ximenynate, potassium lauroyl wheat amino acids, palm glycerides, capryloyl glycine). The active ingredient object of the present study has been developed by Sinerga Research Center Laboratories (Pero, Italy) and is based on patented delivery system [10].

\subsection{Trial Design}

A double blind study was carried out for 4 weeks of treatment, on 22 female healthy informed volunteers, aged between 44 and 64 years old (mean $=54$ ), with low/moderate roughness grade in the area around the eyes. The project was approved by the DermIng Scientific Board. 
The aim of the study was to evaluate by clinical and instrumental evaluations the efficacy and tolerability of a cosmetic treatment in the prevention of the circles under the eyes and blemishes of skin aging on the eye profile $v s$. placebo (same formulation without the active ingredient).

Clinical and instrumental evaluations were performed at baseline and after 2 and 4 weeks of treatment. The study was planned as a double blind half side trial [11-14]. Each product was applied unilaterally (right or left side according to a predetermined randomization list) in the area around eyes, twice a day. Only one volunteer dropped out for reasons unrelated to the study. Therefore, 21 subjects completed the trial. Three visits were carried out during the study: at baseline (T0) and after 2 (T2) and 4 (T4) weeks of treatment.

\subsection{Clinical and Instrumental Evaluation}

To determine the efficacy of the tested treatments, the following clinical/instrumental evaluations) [15] were performed:

- Wrinkles grade (visual score) of the area around the eyes (deep and fine wrinkles) using a reference photographic scale: 0 , no wrinkles; 1 , very mild; 2 , mild; 3 , moderately evident; 4 , evident; 5 , very evident; 6 , marked; 7 , very marked;

- Circles under the eye (visual score): 0, absent, 1, quite visible; 2, visible; 3, marked; 4, very marked;

- Skin dryness (clinical score): 0, extremely hydrated; 1, hydrated; 2, normal; 3, quite dry; 4, dry; 5, very dry;

- Skin smoothness (clinical score): 0, very good; 1, good; 2, moderate; 3, low; 4, very low;

- Visualization of the skin aging grade of the area around the eyes by the Spiderming ${ }^{\circledR}$ graph (DermIng S.r.l. Clinical Research Institute, Monza, Italy), that allowed us to evaluate the results and visualize the effect of the cosmetic products. Taking into account that a smaller area of the graph coincides with a younger skin, the activity of the products in terms of anti-age activity could be quantified;

- Skin electrical capacitance measurement for stratum corneum hydration (corneometry) [16,17]. Skin hydration was measured by the instrument Corneometer CM820 (Courage-Khazaka, Köln, Germany). To reduce the variability of measurements, three repeated measures on the same skin area (periocular area) were performed and their adjusted mean was considered as the real measure value; spectrophotometry [18]. The efficacy of the test product was evaluated with a spectrophotometric measurement (Ocean Optics, Dunedin, FL, USA) of the skin color on the circles under the eyes. Spectrophotometric evaluations employed a spectrophotometer for the spectra of visible, ultraviolet and infrared ( $\lambda$ from 188 to $1100 \mathrm{~nm}$ ) using a tungsten halogen lamp and a deuterium lamp compliant to CIE (Commission Internationale de l'Eclairage). The light source was turned on $30 \mathrm{~min}$ prior to the use of the equipment in order to stabilize the lamp emissions. The inclination of the probe was $90^{\circ}$ on the surface to be examined, on an area of $c a .2 \mathrm{~mm}^{2}$. The wavelength range was $380-780 \mathrm{~nm}$, corresponding to the visible spectrum;

- Photographic documentation and profilometry [19-25] of the wrinkles around the eyes (“crow's feet"). The photographic documention was performed with Primos ${ }^{\circledR}$ compact portable system (GFMesstechnik, Berlin, Germany), a phase-shift rapid in vivo measurement which 
provides high-resolution profilometry of skin surfaces by using phase-shifted light stripes projected. Defining an area within the image and tracing a segment of known length in a defined position across the wrinkle and perpendicular to it, it is possible to calculate the profilometric parameters as follows:

- $R_{\mathrm{a}}$, roughness average parameter which is the arithmetic mean of all ordinates from mean line of profile;

- $R_{\mathrm{t}}$, wrinkles depth mean value;

- $R_{\mathrm{z}}$, wrinkles height mean value;

- $R_{\max }$, maximum wrinkles height;

- $R_{\mathrm{v}}$, maximum wrinkles depth.

The tolerance of the study treatment was evaluated by recording and monitoring any adverse event and by dermatological assessment of objective symptoms at baseline and on each following visit.

Regarding the ophthalmologic tolerance, the following symptoms were recorded in basal conditions and throughout the study period:

- Eyelid signs: erythema, oedema, desquamation;

- Ocular signs: conjunctival injection (redness).

Moreover, at the end of the study, volunteers completed self assessment questionnaire to evaluate product efficacy and the cosmetic acceptability.

\section{Statistical Analysis}

Statistical analysis (S-Plus Insightful Corporation, Seattle, Washington, DC, USA) was carried out on a total of 21 subjects who completed the trial. The data processing was performed as follows [26]:

- Evaluation of each study product vs. basal conditions (T2 and T4 vs. T0);

- Clinical assessment: Friedmann test followed, in case of statistically significant result, by Dunnett test for multiple comparisons;

- Instrumental measurements: analysis of variance (ANOVA) test followed, in case of statistically significant result, by Dunnett test for multiple comparisons;

- Comparison study product vs. placebo time by time;

- Clinical assessment: Wilcoxon test (Rank Sum);

- Instrumental measurements: Student's $t$ test (two-sample $t$ ).

\section{Results}

\subsection{Clinical Evaluation}

Obtained results highlighted as the active product determined, at the end of the study, a clinically and statistically (Dunnett test $p<0.05 \mathrm{~T} 4 v s$. T0) significant improvement $v s$. baseline of the following items:

- Deep wrinkles on lateral corner of the eyes: reduction of the visual score of at least one grade in $52.5 \%$ of treated cases; 
- Fine wrinkles on lateral corner of the eyes: reduction of the visual score of at least one grade in $67 \%$ of subjects;

- Circles under the eyes: reduction of the clinical score of at least one grade in $76 \%$ of volunteers; this reduction was statistically significant in respect to the one obtained for the placebo (Wilcoxon test $p<0.06 \mathrm{~T} 4_{\text {active }} v s . \mathrm{T} 4_{\text {placebo) }}$;

- Skin smoothness: decrease of the clinical score of at least one grade in 53\% of subjects;

- Skin dryness: reduction of the clinical score of at least one grade in $67 \%$ of volunteers, although the statistical significance showed at T2 was not confirmed at T4.

For the placebo, no significant variation of deep and fine wrinkles and of circles under the eyes vs. T0 was found; at the end of the study period a significant improvement (Dunnett test $p<0.05 \mathrm{~T} 4 v s$. T0) of skin smoothness of at least one grade in $57 \%$ of cases was highlighted and, already after 2 week-treatment, a considerable and statistically significant improvement of skin dryness was observed (Dunnett test $p<0.05 \mathrm{~T} 2$ and T4 vs. T0); in particular the improvement of at least one grade of the clinical score was observed respectively in $58 \%$ of treated subjects at $\mathrm{T} 2$ and in $85 \%$ at $\mathrm{T} 4$.

Clinical assessment (mean value) carried out at each visit for both products were summarized in the Spiderming $^{\circledR}$ graph, which underlines the "anti-aging" activity of the active product on the principal signs of skin aging; if a larger graph area corresponds to a state of more advanced skin aging, it is evident that after a 4-week treatment the skin area around the eyes improved in appearance (Figure 1) with a statistically significant reduction of all considered clinical signs (wrinkles, circles under the eyes, skin smoothness, skin dryness scores - mean value). In addition, for the circles under the eyes, this reduction was statistically significant $v s$. placebo, a sign that the circles under the eyes treated with the active product are less marked and less evident than those treated with the placebo product (Figure 2).

Figure 1. Clinical assessment (Spiderming ${ }^{\circledR}$ graph) of the active product.

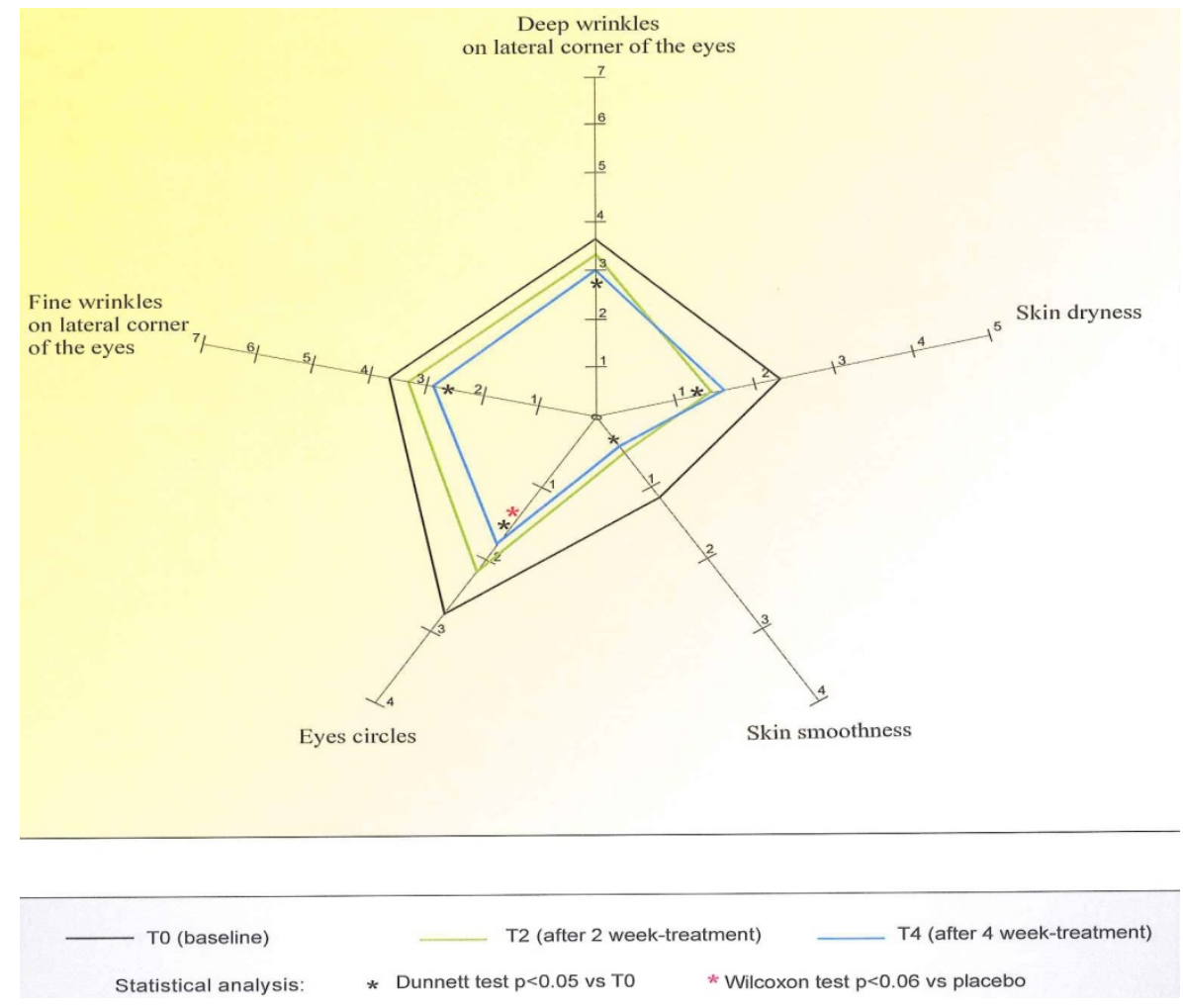


Figure 2. Clinical assessment (Spiderming ${ }^{\circledR}$ graph) of the placebo.

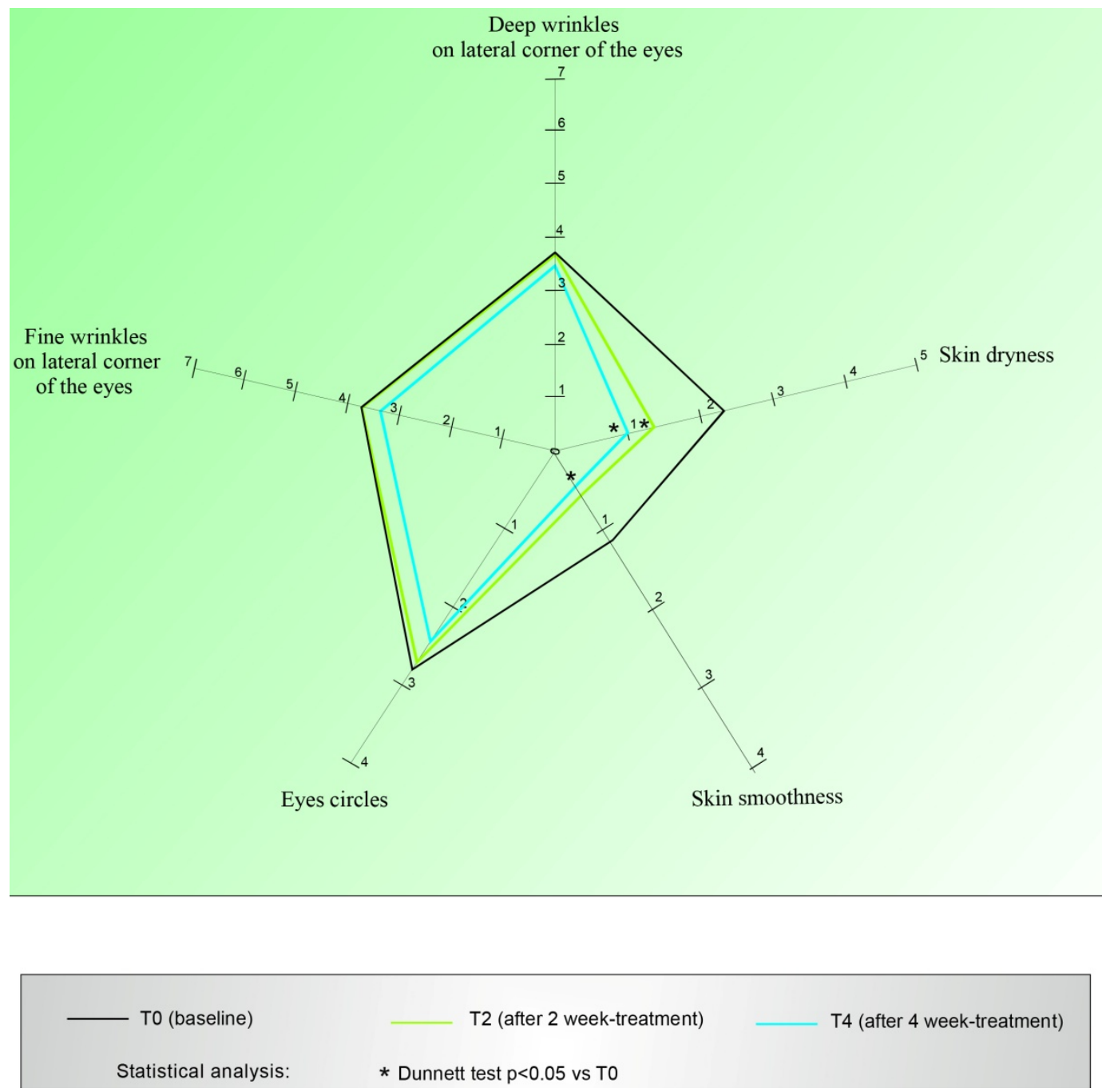

\subsection{Instrumental Evaluation}

As highlighted by the clinical assessment, the placebo determined a statistically significant improvement (Dunnett test $p<0.05 \mathrm{~T} 4 \mathrm{vs}$. T0) of skin hydration mean value corresponding to an increasing percentage of $6.1 \%$ vs. baseline. In contrast, for the active product no clinical significant variation of skin electrical capacitance was found.

Although no statistically significant variation of the total visible spectrum area $(\lambda 380-780 \mathrm{~nm})$ for both products was detected at any study time, it is important to note for the active product at T2 a clinical important reduction of the total spectrum area of $6 \% v s$. baseline (from $\mathrm{T}_{\text {mean value }}=6683.8$ to $\mathrm{T} 22_{\text {mean value }}=6285.2$ ). This result was probably determined by an initial draining activity of the product on the interstitial liquids accumulated which lead to an initial vasoconstriction. Otherwise, at the end of 4 week-treatment, the active product induced a clinically relevant increase of the total spectrum area corresponding to a reduction of the color of the circles under the eyes of $7.7 \%$ (from $\mathrm{T} 0_{\text {mean value }}=6683.8$ to $\left.\mathrm{T} 4_{\text {mean value }}=7195.7\right)$, index of an improvement of the skin brightness and of the general conditions of skin vascularisation. As confirmed by a clinical assessment of the circles under the eyes, this activity was shown to be more marked than the one highlighted for the placebo product 
(7.7\% active $v$ s. $5.4 \%$ placebo), even if no statistically significant difference between the two products was found (Figure 3).

Figure 3. Spectrophotometric measurements of the skin color of the circles under the eyes. Active product vs. placebo.

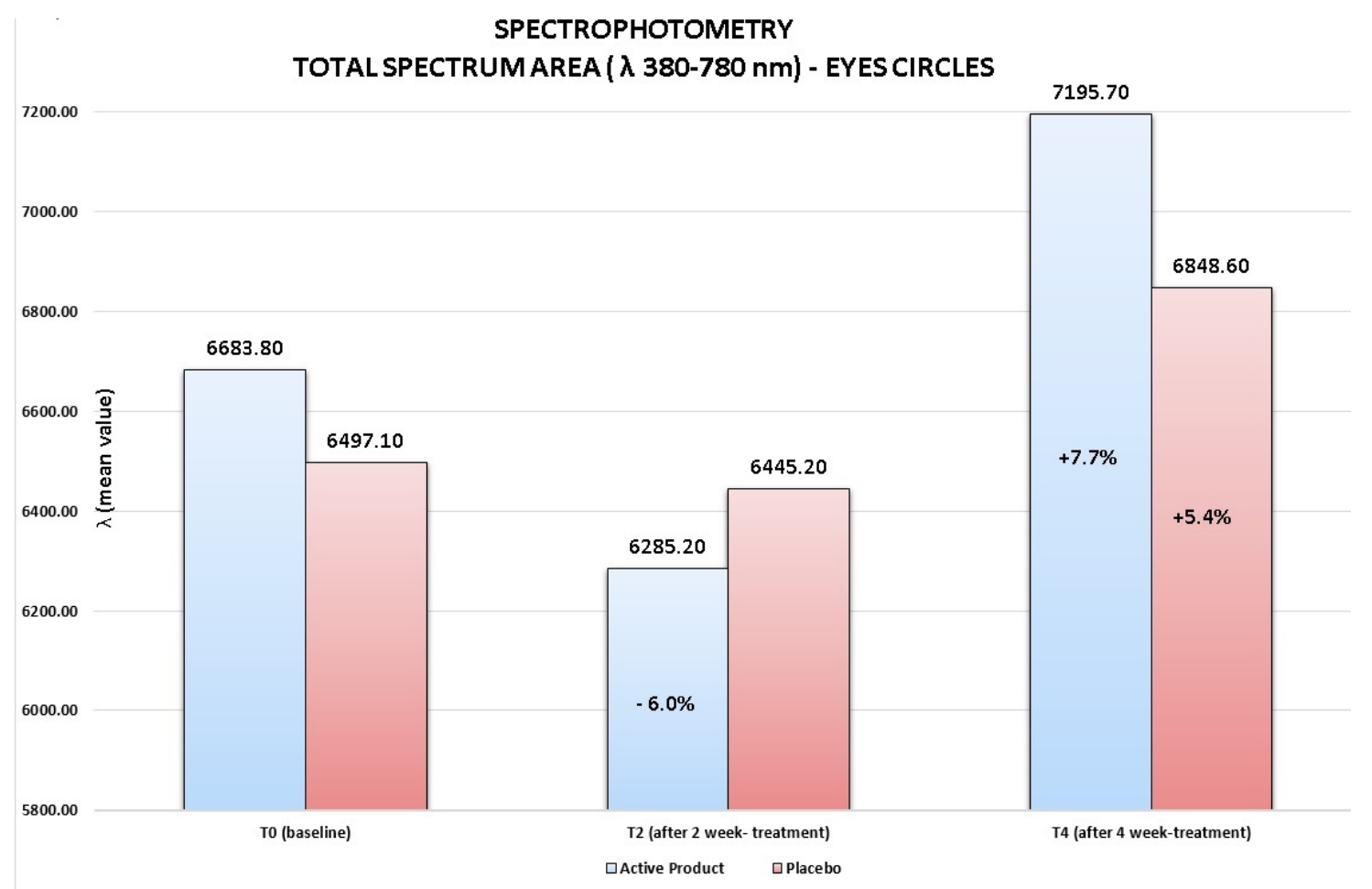

Image analysis on the wrinkles around the eyes (crow's feet area), performed on the pictures taken by Primos ${ }^{\circledR}$ compact portable device, highlighted for both products a clinically noticeable reduction of the principal profilometric parameters, which indicates that wrinkles at the end of the trial were less deep and marked (Figure 4). In particular although no statistically significant variation was showed, the following reduction percentages $v s$. baseline (T0) were obtained (Figures 5 and 6):

1. Active product:

- $-11 \%$ at T2 and $-8 \%$ at T4 for $R_{\mathrm{a}}$ parameter, that represents the average roughness;

- $-13 \%$ at T2 for $R_{\mathrm{t}}$ and $R_{\mathrm{z}}$ parameters that represent respectively wrinkles average depth and average height;

- $-16.3 \%$ at T2 (Dunnett test $p<0.05$ ) and $-10 \%$ at T4 for $R_{\max }$ parameter that represents the wrinkles maximum height;

- $-6 \%$ at $\mathrm{T} 2$ and $-4 \%$ at $\mathrm{T} 4$ for $R_{\mathrm{v}}$ parameter that represents the wrinkles maximum depth.

2. Placebo:

- $-13 \%$ at T2 for $R_{\mathrm{a}}$;

- $-19 \%$ at T2 and $-14 \%$ at T4 for $R_{\mathrm{t}}$;

- $-12 \%$ at T2 and $-9 \%$ at T4 for $R_{\mathrm{z}}$;

- $-16 \%$ at T2 and $-9 \%$ at T4 for $R_{\max }$;

- $-10 \%$ at T2 and $-4 \%$ at T4 for $R_{\mathrm{v}}$. 
Figure 4. Volunteer $n$. 3. 3D photos by $\operatorname{Primos}^{\circledR}$ System at baseline, after 2 and 4 week-treatment.

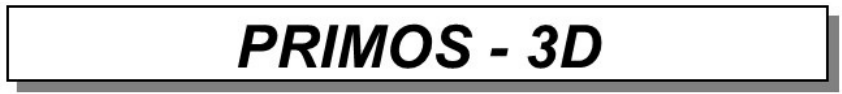

Volunteer n. 3 - Active Product

To
(baseline)

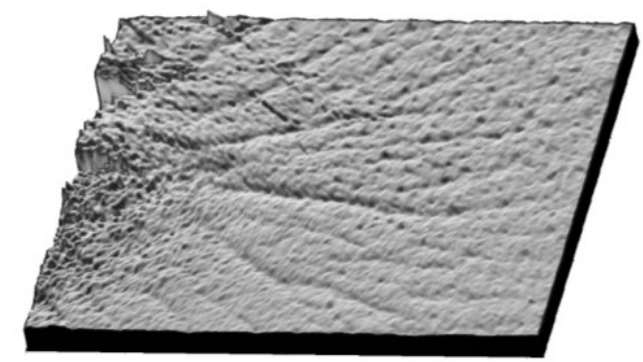

T2

(after 2 week - treatment)

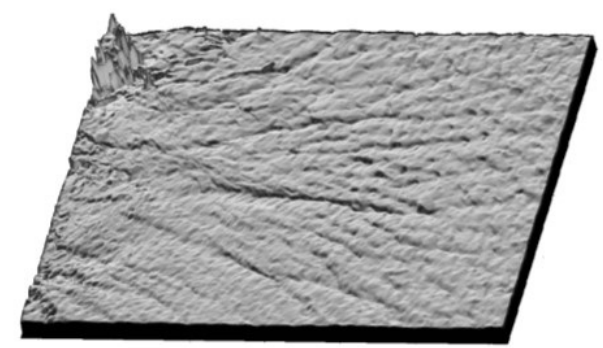

T4

(after 4 week - treatment)

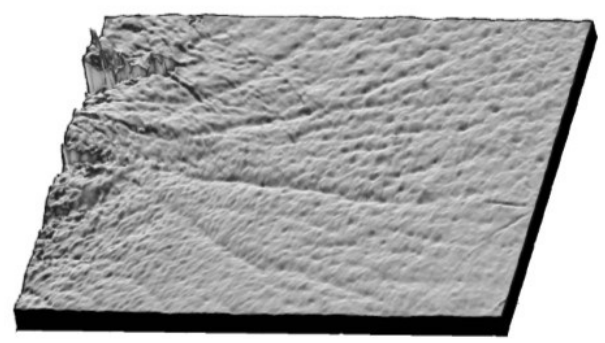

\subsection{Tolerability}

The clinical evaluation performed bilaterally in the eyelids (erythema oedema, desquamation) and the conjunctiva (conjunctival injection) did not show any significant reaction; no adverse event related to the study products occurred during the trial and the good tolerance of the study products was also confirmed by the volunteers' judgment. After the first products application, some subjects described the appearance of mild skin erythema ( 3 subjects for the active product and 4 subjects for the placebo) and/or mild pinching/burning sensation ( 8 subjects for the active product and 6 subjects for the placebo) which resolved in a few minutes and only in one case was followed by skin dryness (one subject for both products) or skin desquamation (one subject for the placebo). 
Figure 5. Active product: profilometric measurements, variation vs. baseline.

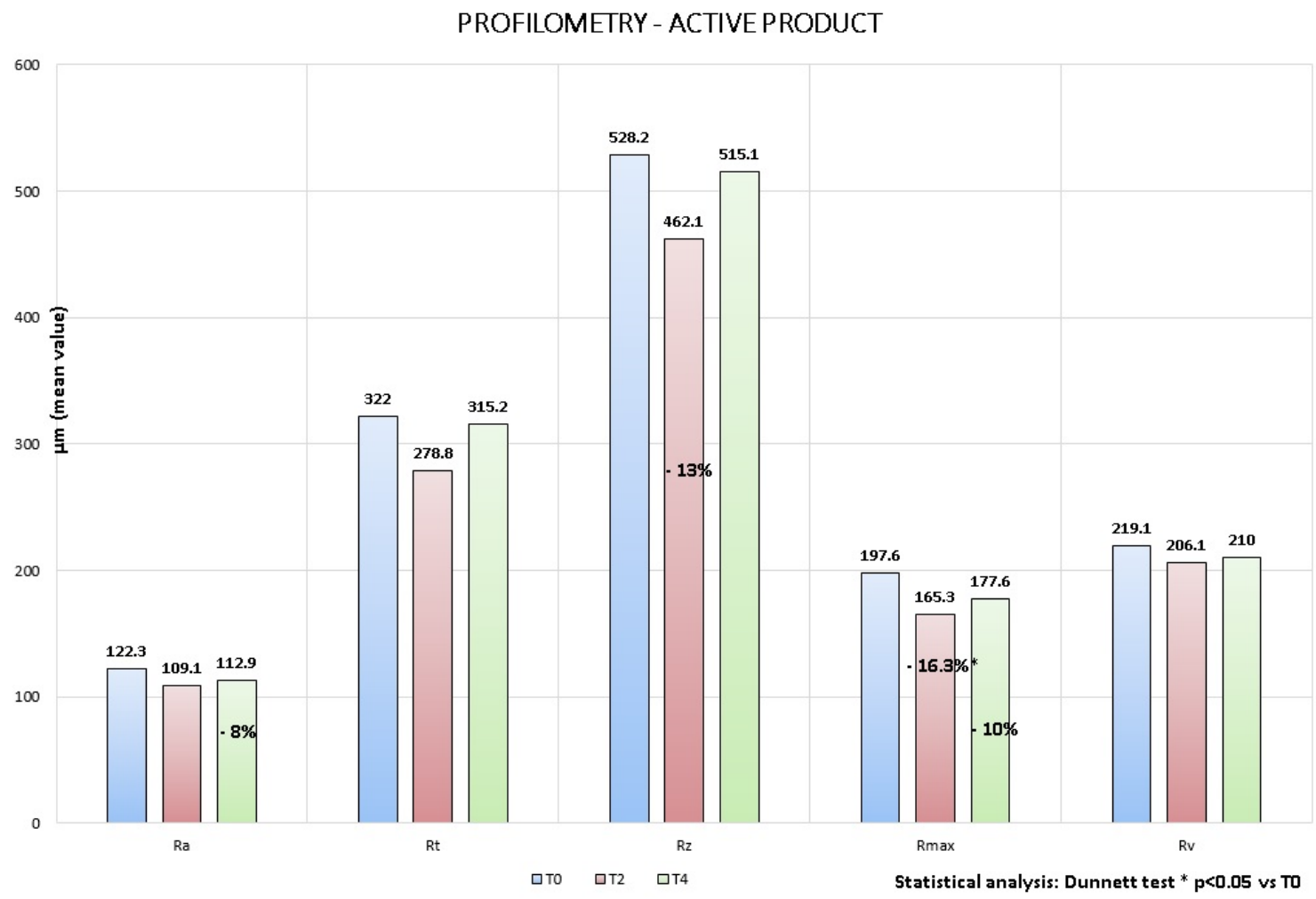

Figure 6. Placebo: profilometric measurements, variation vs. baseline.

PROFILOMETRY - PLACEBO

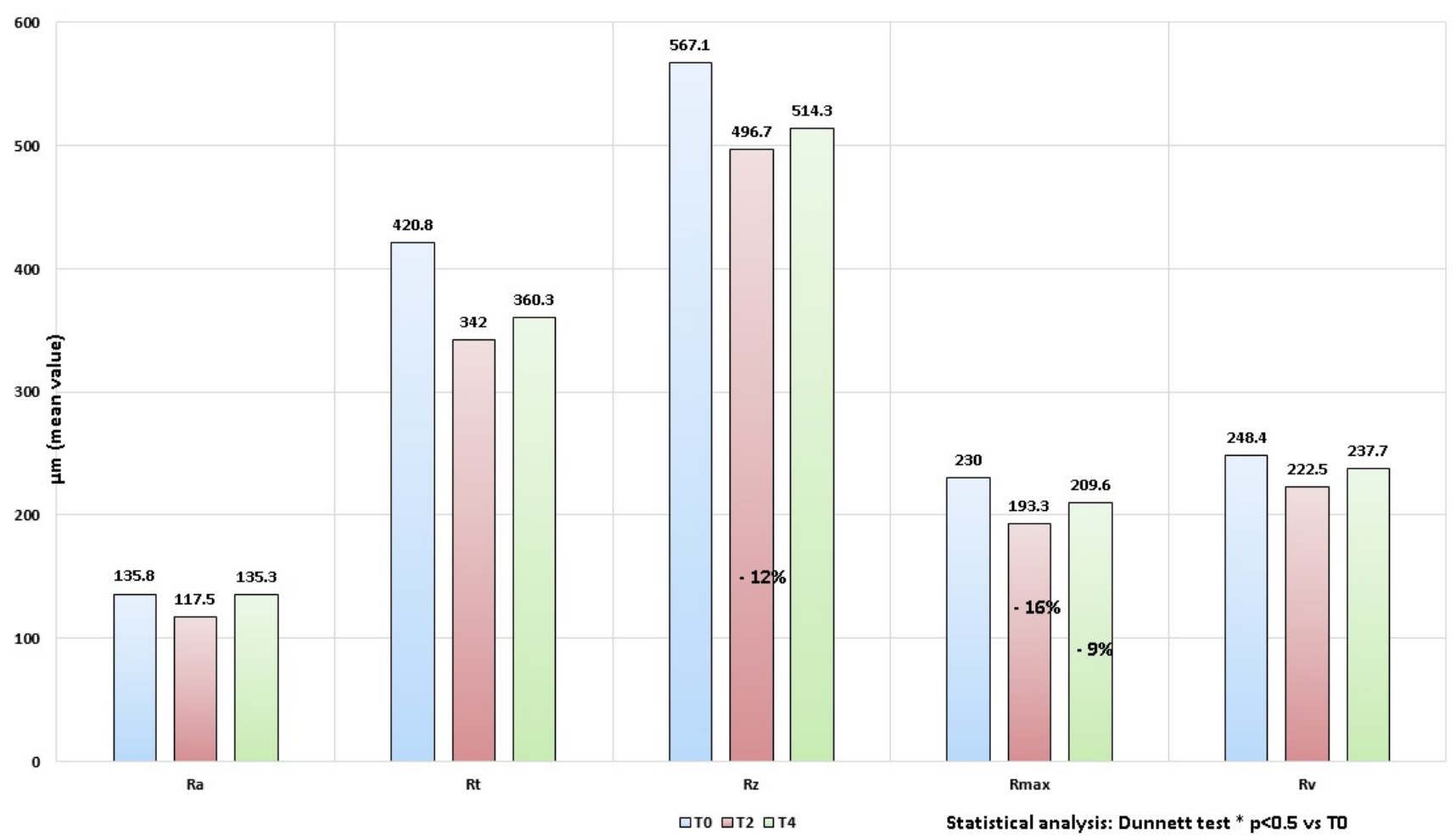




\subsection{Self Assessment Evaluation}

The majority of the subjects noticed the efficacy of the active product; in particular $71.4 \%$ of subjects described an improvement of deep wrinkles (57.1\% medium, 9.5\% marked and 4.8\% very marked) and $76.2 \%$ an improvement of fine and superficial wrinkles $(57.1 \%$ medium, $14.3 \%$ marked and $4.8 \%$ very marked), while $71.4 \%$ of volunteers observed a reduction of eyes puffiness $(47.6 \%$ medium and $23.8 \%$ marked) and $66.6 \%$ of circles under the eyes $(33.3 \%$ medium, $19 \%$ marked and $14.3 \%$ very marked) that appeared at the end of the study more stretched and less visible. Moreover, $80.9 \%$ of volunteers noticed an improvement of skin dryness $(47.6 \%$ medium, $23.8 \%$ marked and $9.5 \%$ very marked). The efficacy of the placebo product was noticed by an important, but less consistent percentage of subjects.

The cosmetic acceptability of both products was generally excellent. In particular, a high percentage of volunteers appreciated the products (active and placebo) color (good-excellent respectively for 95\% and $100 \%$ of subjects), perfume (good-excellent for $76 \%$ of subjects), consistency (good-excellent respectively for $90 \%$ and $86 \%$ of subjects), spreadability (good- excellent respectively for $100 \%$ and $86 \%$ of subjects), as well as absorption (good-excellent respectively for $90 \%$ and $71 \%$ of subjects), effect on the skin after application (good-excellent respectively for $90 \%$ and $76 \%$ of subjects) and absence of product residues on the treated areas (good-excellent respectively for $100 \%$ and $86 \%$ of subjects).

\section{Discussion}

Midfacial aging is a multifactorial, three dimensional process that involves volume loss, volume descent, and skin alterations. In the periocular area, age-related changes include fine wrinkles in the lower eyelids and lateral orbital area, dyschromias, and alterations in texture and pigmentation. The subcutaneous vasculature loses its papillary organization, the dermal/epidermal interface flattens, and the dermis and subcutaneous tissues atrophy [27]. Dark circles under the eyes are caused by multiple etiologic factors that include periorbital edema and superficial location of vasculature [28]. Therefore, treatment of the periocular area should be oriented to the improvement of skin microcirculation.

\section{Conclusions}

The study product Ethyl Ximenynate (International Nomenclature of Cosmetic Ingredients: ethyl ximenynate, potassium lauroyl wheat amino acids, palm glycerides, capryloyl glycine), has been demonstrated to be effective in fighting eyes swelling.

After the 4-weeks treatment, the skin improved appearance due to the general decrease of all clinical scores (eyes puffiness, skin smoothness, roughness and dryness).

The profilometric results confirm the anti-wrinkles activity of the active product and its lifting action, while the spectrophotometer shows the mild decongestant effect on dark circles.

The improvement of skin microcirculation and capillary resistance and vascular tone in the eye area supports the clinical evidence of an improvement in parameters on skin aging and photodamage measured in the study. 


\section{Acknowledgments}

The study was financially supported by Sinerga SpA.

\section{Conflicts of Interest}

The study was supported by Sinerga SpA, the company that developed the active ingredient used in the trial.

\section{References}

1. Love, L.P.; Farrior, E.H. Periocular anatomy and aging. Facial Plast. Surg. Clin. North Am. 2010, $18,411-417$.

2. Galatoire, O.; Morax, S. Periocular aging: Physiopathogenesis, clinical aspect, and treatment. Ann. Dermatol. Venereol. 2009, 136, 137-141.

3. Bombardelli, E.; Spelta, M. Phospholipid-polyphenol complexes-A new concept in skin care ingredients. Cosmet. Toilet. 1991, 106, 69.

4. Eliton, C.; Davindsonl, B.C. A comparison of the lipid and fatty acid profiles from the kernels of the fruit (nuts) of Ximenia caffra and Ricinodendron rautanenii from Zimbabwe. Ind. Crops Prod. 2008, 27, 29-32.

5. Croft, K.D.; Beilin, L.J.; Ford, G.L. Differential inhibition of thromboxane B2 and leukotriene B4 biosynthesis by two naturally occurring acetylenic fatty acids. Biochim. Biophys. Acta 1987, 921, $621-624$.

6. Desai, V.B.; Shankaranarayana, K.H. On the utilization aspects of sandal seed oil. Res. Ind. 1990, 35, 232-233.

7. Liu, Y.; Longmore, R.B. Dietary sandalwood seed oil modifies fatty acid composition of mouse adipose tissue, brain, and liver. Lipids 1997, 32, 965-969.

8. Nugteren, D.H.; Christ-Hazelhof, E. Naturally occuring conjugated octadecatrienoic acids are strong inhibitors of prostaglandin biosynthesis. Prostaglandins 1987, 33, 403-417.

9. Cristoni, A.; Guglielmini, G.; Stucchi, P.; Bouet, A. An unsaturated fatty acid from traditional African cosmesis. In Proceedings of Cosmetics USA, New York, NY, USA, 16-18 November 1999.

10. Comini, M.; Lenzini, M.; Guglielmini, G. Nanoemulsions Comprising Lipoaminoacids and Monoglycerides, Diglycerides and Polyglycerides of Fatty Acids. Ital. Patent MI2005A000218, 24 August 2006.

11. ICH Harmonised Tripartite Guideline. Available online: http://www.ich.org/fileadmin/Public Web_Site/ICH_Products/Guidelines/Efficacy/E6_R1/Step4/E6_R1_Guideline.pdf (accessed on 14 May 2014).

12. The World Medical Association. Available online: http://www.wma.net/en/30publications/ 10policies/b3/ (accessed on 14 May 2014).

13. Rieger, M.M.; Battista, G.W. Some experiences in the safety testing of cosmetics. J. Soc. Cosmet. Chem. 1964, 15, 161-172.

14. Curry, A.S.; Gettings, S.D.; McEwen, G.N. CTFA Safety Testing Guidelines; The Cosmetic, Toiletry and Fragrance Association: Washington, DC, USA, 1991. 
15. Camarosa, J.G.; Anthoine, P.; Tribo Boixareu, M.J.; Serra Baldrich, E.; Aubert, L. Demonstration of the anti-wrinkle efficacy of a cosmetic product. Correlation between clinical observations and instrumental methods. J. Appl. Cosmetol. 1997, 15, 13-20.

16. Elsner, P.; Berardesca, E.; Maibach, H. Bioengineering of the Skin: Water and the Stratum Corneum; CRC Press: Boca Raton, FL, USA, 1994; pp.171-175.

17. Tagami, H. Evaluation of skin surface hydration in vivo by electrical measurements. J. Invest. Dermatol. 1980, 75, 500-507.

18. Zomios, G.; Bykowski, J.; Kollias, N. Skin melanin, hemoglobin and light scattering properties can be quantitatively assessed in vivo using diffuse reflectance spectroscopy. J. Invest. Dermatol. 2001, 117, 1452-1457.

19. Cook, T.H. Profilometry of the skin: A useful tool for the substantiation of cosmetic efficacy. J. Soc. Cosmet. Chem. 1980, 31, 339-359.

20. Hatzis, J. Skin surface profile technique and its applications. Int. J. Cosmet. Sci. 1991, 13, 281-291.

21. Jaspers, S.; Hopermann, H. Rapid in vivo measurement of the topography of human skin by active image triangulation using a digital micromirror device. Skin Res. Technol. 2006, 5, 195-207.

22. Leveque, J.L. EEMCO guidance for the assessment of skin topography. J. Eur. Acad. Derm. Ven. 1999, 12, 103-114.

23. Wilhelm, K.P.; Elsner, P.; Berardesca, E.; Maibach, H.I. Bioengineering of the Skin: Skin Surface Imaging and Analysis; CRC Press: Boca Raton, FL, USA, 1997; pp. 129-143.

24. Frankowsky, G.; Hainich, R. DLP-Based 3D metrology by structured light or projected fringe technology for life sciences and industrial metrology. Proc. SPIE 2009, 2009, doi:10.1117/12.807709.

25. Corcuff, P.; Chatenay, F.; Leveque, J.L. The impact of aging on the microrelief of peri-orbital and leg skin. J. Soc. Cosmet. Chem. 1987, 82, 145-152.

26. Sachs, L. Applied Statistics: A Handbook of Techniques; Springer: Heidelberg, Germany, 1981; pp. 536-539.

27. Wulc, A.E.; Sharma, P.; Czyz, C.N. The Anatomic Basis of Midfacial Aging. In Midfacial Rejuvenation; Wulc, A.E., Sharma, P., Czyzet, C.N., Eds.; Springer Science Business Media: New York, NY, USA, 2012; pp.15-28.

28. Freitag, F.M.; Cestari, T.F. What causes dark circles under the eyes? J. Cosmet. Dermatol. 2007, 6, 211-215.

(C) 2014 by the authors; licensee MDPI, Basel, Switzerland. This article is an open access article distributed under the terms and conditions of the Creative Commons Attribution license (http://creativecommons.org/licenses/by/3.0/). 